\title{
A comparative evaluation of three methods for the rapid diagnosis of cryptococcal meningitis (CM) among HIV-infected patients in Northern Malawi
} Master R.O. Chisale ${ }^{1,2,3}$, Dikani Salema ${ }^{1,4}$, Frank Sinyiza ${ }^{1}$, Judith Mkwaila ${ }^{1}$, Pocha Kamudumuli ${ }^{5}$, Hsin-yi Lee ${ }^{2,3.6}$

1. Mzuzu Central Hospital, Ministry of Health, Mzuzu, Malawi

2. Luke International, Mzuzu, Malawi

3. Department of Biomedical Sciences, Mzuzu University, Mzuzu, Malawi

4. University of Malawi, College of Medicine, Blantyre, Malawi

5. Malawi Lab Project, University Research Co., LLC, Lilongwe, Malawi

6. Pingtung Christian Hospital, Pingtung, Taiwan

\begin{abstract}
Introduction
Abstract

Cryptococcal meningitis (CM) is the most common systemic fungal infection in patients with HIV infection. Rapid diagnosis and timely initiation of antifungal therapy are key to reducing mortality rate associated with CM. This study aims to evaluate the ability of four different diagnostic tests (Gram stain, India ink, and two types of commercial lateral flow assay [LFA]) to identify CM-positive patients and to compare the sensitivity and specificity of these tests.

Methods

This was a prospective cross-sectional study on diagnostic tests accuracy conducted in Northern Malawi. The target population was HIV-infected adult patients presenting with features of meningitis. Four types of diagnostic tests were conducted: India ink, Gram stain, and two types of commercial lateral flow assay (LFA) (Immy, Inc., OK, USA and Dynamiker Biotechnology (Tianjin) Co., Ltd), Singapore). Culture was conducted as the reference standard.

Results

A total of 265 samples were collected. The rate of positive CM detection ranged from $6.4 \%$ (using India ink) to $14.3 \%$ (using LFA). India ink exhibited the lowest sensitivity of 54.8\% (95\% confidence interval [CI]: 36.0\%-72.7\%), followed by Gram stain (61.3\%; $95 \%$ CI: $42.2 \%-78.2 \%)$. The Dynamiker LFA exhibited the highest sensitivity of $100.0 \%(95 \%$ CI: 90.0\%-100.0\%) but a lower specificity $(97.0 \%$; 93.9\%-98.8\%) compared to the Immy LFA (98.3\%; 95\% CI: 95.7\%-99.5\%).

Conclusion

LFA diagnostic methods have the potential to double the detection rate of CM-positive patients in resource-limited countries such as Malawi. As such, LFAs should be considered to become the main diagnostic tests used for CM diagnostics in these countries. Our data indicate that LFAs may be the best method for diagnosing CM and exhibits the highest diagnostic accuracy as it has shown that it outperforms cell culture, the current gold standard.

Key Words: Diagnostic methods, cryptococcal meningitis, evaluation, comparison, fungal infection
\end{abstract}

\section{Introduction}

Cryptococcal meningitis $(\mathrm{CM})$ is the most common systemic fungal infection in patients with HIV infection. Globally, there are an estimated 181,100 estimated deaths from CM, with $75 \%$ of the deaths occurring in sub-Saharan Africa $(\mathrm{SSA})^{1}$. Despite the increased coverage of antiretroviral treatment (ART) programs in high burden countries, the case fatality rate of CM remains high at 35\%-65\% in $\mathrm{SSA}^{2-4}$. In Malawi, studies have reported that more than $40 \%$ of patients diagnosed with $\mathrm{CM}$ die within 4 weeks of the diagnosis, and that $55 \%-58 \%$ die by 10 weeks $^{5,6}$. Consequently, early diagnosis and the timely initiation of antifungal therapy is essential if we are to reduce morbidity and mortality from cryptococcal disease.

The clinical presentations of $\mathrm{CM}$ are often indistinguishable from other causes of meningitis. As such there is a need for a more accurate and robust diagnostic test for the diagnosis of $\mathrm{CM}^{7}$. The laboratory diagnosis of $\mathrm{CM}$ includes direct visualization of cryptococci via microscopy, culture of the organism and/or the detection of cryptococcal antigens in cerebrospinal fluid (CSF). Even though India ink is rated poorly in terms of diagnostic ability for $\mathrm{CM}$, and with regards to sensitivity and specificity ${ }^{8-11}$, evidence indicates that India ink stain is still widely used for the detection of cryptococci in CSF, particularly in resource-limited countries such as Malawi ${ }^{12}$.

Although cell culture is relatively slow, it is still considered to represent the gold standard for CM diagnosis. Antigen detection is rapid, and is associated with high sensitivity (93\%-100\%) and specificity (93\%-98\%); however, this form of detection is not available in low-resource countries due to its excessive costs ${ }^{10}$. Therefore, microscopy continues to be widely adopted as a rapid, cheap and reliable diagnostic method in these settings.

There are few published data on the utility of the Gram stain for the direct detection of cryptococci in CSF samples, particularly in patients infected with HIV. Furthermore, there is little published data available that compares microscopic and antigen-based CM diagnostic methods, particularly in resource-constrained settings such as Malawi. We therefore undertook a comparative evaluation of Gram stain, India ink stain, and two forms of lateral flow assay (LFA) (Immy and Dynamiker), for the rapid diagnosis of cryptococcal meningitis in HIV-infected patients in Mzuzu, Malawi. 
The main objectives of the study were to: (1) establish the detection rate of positive-CM patients among HIV-infected patients presenting with features of meningitis in Mzuzu Central Hospital by using different diagnostic methods, and (2) compare the sensitivity and specificity of the Gram stain, India ink and two LFA tests for the diagnosis of CM.

\section{Methods \\ Study design}

This was a prospective cross-sectional study relating to the accuracy of diagnostic tests for CM conducted at Mzuzu Central Hospital, Northern Malawi.

\section{Setting}

Mzuzu Central Hospital is a government tertiary hospital located in the northern part of Malawi that serves a population of about 2.5 million $^{13}$. As a tertiary institution, this hospital is a referral site for all secondary and private hospitals in northern Malawi. The hospital also serves as a secondary health care facility for Mzimba North District due to the lack of a district hospital.

\section{Participants}

This study targeted HIV-infected patients in the hospital. The specific inclusion criteria were males and females aged 18 years or more, presenting with features of meningitis (fever, headache, confusion, neck stiffness, photophobia and vomiting). Potentially eligible participants were identified by clinicians in the outpatient department and inpatient wards. Patients were excluded if they refused to provide consent and/or were taking fungal treatment, or had any history of fungal treatment.

\section{Sampling method}

This was a hospital-based study and we considered the sample size to consist of all patients who attended the hospital during the study period (10 months) who fulfilled the inclusion criteria. Patients with fever, headache, confusion, neck stiffness, photophobia and vomiting, and who were also HIV-positive were included in the study. The participants were recruited in a consecutive series until a sample size of 265 was reached; this sample size met the minimum requirement for this type of study, as indicated in the literature ${ }^{14-16}$.

\section{Laboratory test methods}

Cryptococcus species were identified by morphological, biochemical and serological characteristics. Four types of diagnostic tests were conducted on the samples, including: the India ink test, Gram stain, and two types of commercial LFAs (Immy, Inc. OK, USA; Dynamiker Biotechnology (Tianjin) Co., Ltd). CSF was collected from participants following aseptic standard procedures used in the hospital. Microscopy techniques were then used to carry out morphological tests to identify cryptococcus directly from the CSF using India ink staining and Gram staining; these tests were carried out in accordance with established standard operating procedures $(\mathrm{SOPs})^{14}$. In the India ink test, the polysaccharide capsule does not take up the stain, resulting in the appearance of a refractile large white circle against a dark background. For the Gram stain, cryptococci appear as single, budding, thin-walled, oval to round cells containing gram-positive granular inclusions, surrounded by a capsule that varies in size and appears to be gram-negative. Characteristically, the attachment between the parent and daughter cell is extremely narrow, and a lattice formation is evident within a cluster of cryptococci. Immunochromatographic assays were employed for the serological detection of cryptococcal capsular polysaccharide antigen in CSF. This was performed in accordance with the manufacturer's recommendations. An immunochromatographic assay allows the detection of a target analyte in a specific sample. The selection of the commercial platform was based on the manufacturer's claims relating to high specificity, sensitivity and the availability of the test kits within the country.

The culture method was used as a reference standard; this methodology represents the gold standard for CM diagnosis at present. CSF sediments were cultured on Sabouraud dextrose agar (SDA) for 48-72 hours according to an established SOP (MZCH317) at Mzuzu Central Hospital Laboratory. Sabouraud media supports fungal growth. We also added a broad-spectrum antibiotic (chloramphenicol or tetracycline) to the media to inhibit the growth of grampositive and gram-negative bacteria. Further identification was based on biochemical tests, mainly Niger seed agar and urease test production. The process used for sample collection and testing is shown in Figure 1.

\section{Quality assurance}

Culture media, gram reaction and the potency of antimicrobial discs were checked against standardized reference strains of Escherichia coli (ATCC 25922) and Staphylococcus aureus (ATCC 25923). Mzuzu Central Hospital Laboratory, Microbiology Department participates in the UK NEQAS and Zimbabwe National Quality Assurance Programme (ZIMQAP) external quality assurance schemes. Both positive and negative controls were also included on a weekly basis for the LFA and on a daily basis for microscopic tests and culturing.

\section{Data variables and collection}

A case report form was developed to capture data variables related to patient demographic characteristics, chief complaints and symptoms, and HIV status. Laboratory test results were extracted from the log book in the Microbiology Department with a structured proforma. Patient privacy and confidentiality was guaranteed as numbers were used for the identification of participants instead of names. Electronic data were protected using a password known only to the investigators. Data collection was performed from May 2017 to July 2018.

\section{Statistical analysis}

Data recorded on the case report form and structured proforma were subsequently entered into a spreadsheet. Data management and analysis were performed using Microsoft Excel. The 95\% confidence intervals (CIs), which are important for diagnostic accuracy, were determined using MedCalc software ${ }^{15}$

\section{Ethics approval}

Permission to carry out the study was obtained from the hospital management research and publication committee. Ethical approval was obtained from the Malawi National Health Science Research Committee in Lilongwe, Malawi (approval number: NHSRC \#17/01/1718).

\section{Results}

A total of 265 samples were collected from eligible participants. All samples were tested using the four diagnostic methods and by routine cell culture (Figure 2). Among the 


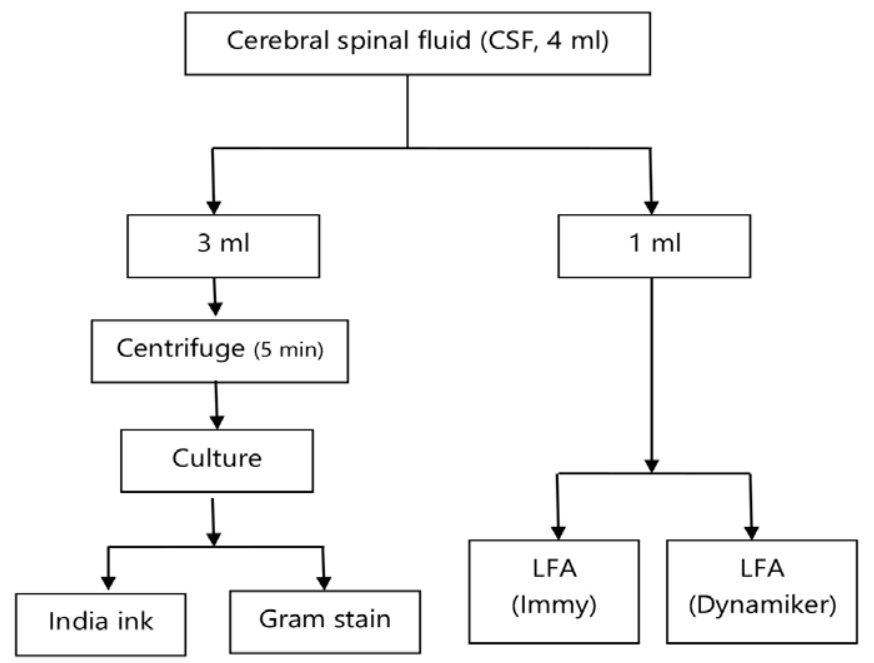

LFA, lateral flow assay.

Figure 1: Sample flow analysis

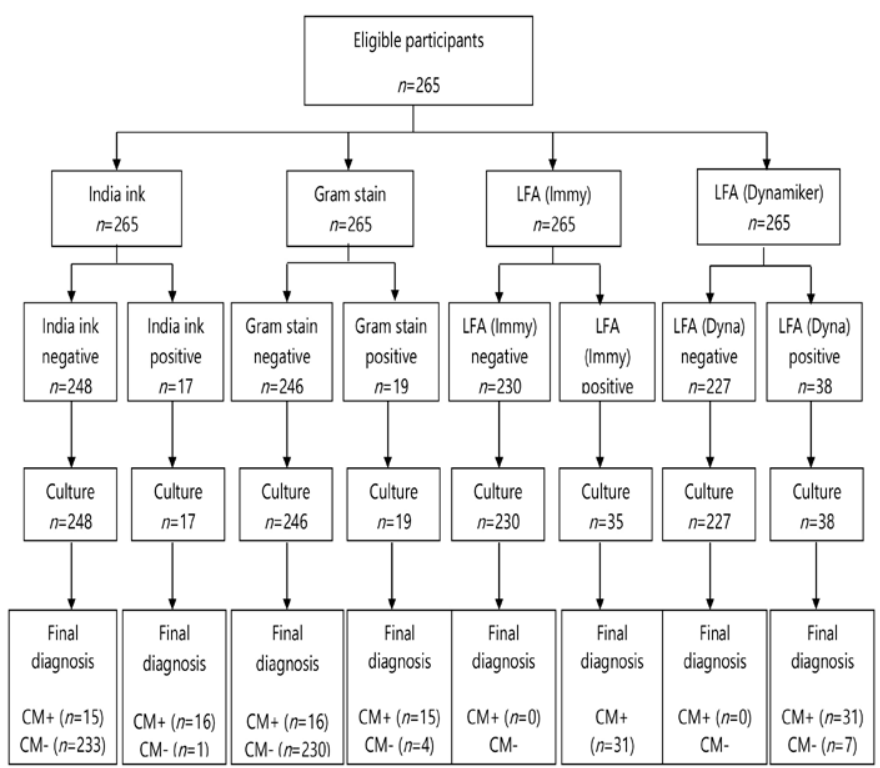

$C M$, cryptococcal meningitis; LFA, lateral flow assay.

Figure 2: Flow of participant (Laboratory results based)

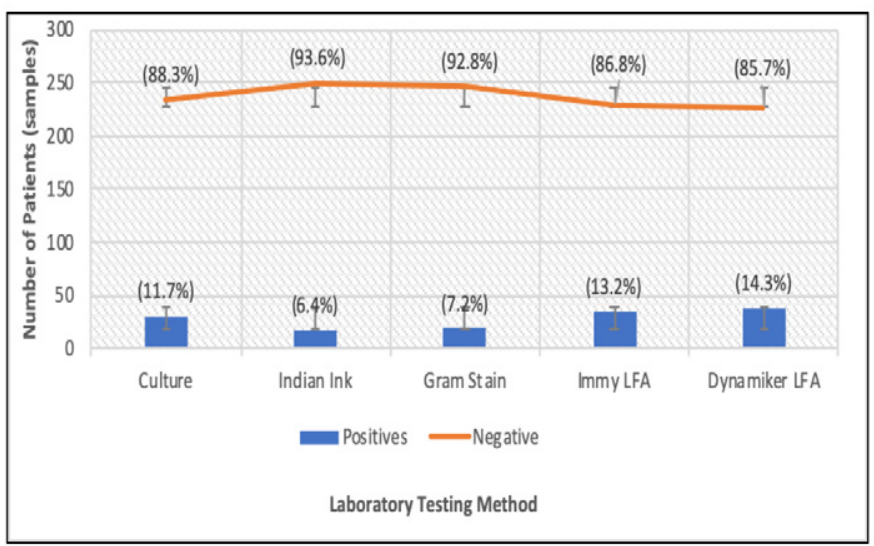

Figure 3: The rate of positive-CM detection by different laboratory diagnostic methods.

study participants, there were more male than females (55.5\% and $45.5 \%$, respectively), and the majority were between 30 and 39 years old (Table 1).
Figure 3 shows the rate of positive-CM identification according to the different diagnostic tests. The rate of positive-CM identification ranged from $6.4 \%$ (using India ink) to $14.3 \%$ (using LFA). Both LFA tests identified more positives than the conventional culture method, our reference standard. The Dynamiker LFA produced the highest rates of positivity, while India ink produced the lowest rates of positivity, identifying only approximately half of the number of cases as the culture method.

\section{Table 1. Demographic characteristics}

\begin{tabular}{lccc}
\hline Age (years) & $\begin{array}{c}\text { Female } \\
n(\%)\end{array}$ & $\begin{array}{c}\text { Male } \\
n(\%)\end{array}$ & $\begin{array}{c}\text { Total } \\
n(\%)\end{array}$ \\
\hline$<20$ & $9(7.6)$ & $8(5.4)$ & $17(6.4)$ \\
$20-29$ & $35(29.7)$ & $29(19.7)$ & $64(24.2)$ \\
$30-39$ & $34(28.8)$ & $49(33.3)$ & $83(31.3)$ \\
$40-50$ & $28(23.7)$ & $41(27.9)$ & $69(26.0)$ \\
$>50$ & $12(10.2)$ & $20(13.6)$ & $32(12.1)$ \\
Total & $118(100.0)$ & 147 & $265(100.0)$ \\
\hline
\end{tabular}

Table 2. Comparison of the sensitivity, specificity, and accuracy, of four laboratory diagnostic methods for cryptococcal meningitis

\begin{tabular}{llll}
\hline Test method & $\begin{array}{l}\text { Sensitivity, } \\
\%(95 \% \mathrm{Cl})\end{array}$ & $\begin{array}{l}\text { Specificity, } \\
\%(95 \% \mathrm{Cl})\end{array}$ & $\begin{array}{l}\text { Accuracy, } \\
\%(95 \% \mathrm{Cl})\end{array}$ \\
\hline India ink & $54.8(36.0-72.7)$ & $100.0(98.5-100.0)$ & $95.0(91.7-97.2)$ \\
Gram stain & $61.3(42.2-78.2)$ & $100.0(98.5-100.0)$ & $95.7(92.6-97.8)$ \\
$\begin{array}{l}\text { Immy LFA } \\
\begin{array}{l}\text { Dynamiker } \\
\text { LFA }\end{array}\end{array}$ & $100.0(90.0-100.0)$ & $98.3(95.7-99.5)$ & $98.5(96.2-99.6)$ \\
\hline
\end{tabular}

A comparison of the diagnostic methods with respect to sensitivity, specificity and accuracy, is shown in Table 2. India ink and Gram stain were associated with the lowest sensitivity $(54.8 \%$ and $61.3 \%)$ but the highest specificity $(100 \%)$ in the diagnosis of CM. Both LFA tests showed $100 \%$ sensitivity and high specificity of up to $98.3 \%$, with the Immy LFA exhibiting the highest level of accuracy (98.5\%).

\section{Discussion}

A positive fungal culture is the gold standard for the diagnosis of cryptococcal infection. Previous research has shown that samples of CSF show fungal growth in almost all cases of $\mathrm{CM}^{10,12,16}$. However, due to problems associated with delay and the critical demand of expertise, fungal culture may not be the most favourable diagnostic method for the clinical management of CM. Furthermore, in situations like Malawi, in which the burden and prevalence of CM is low, there is a possibility that the culture method may not identify every case of CM.

In this study, the Gram stain was found to be marginally more sensitive $(61.3 \%)$ than the India ink stain (54\%), although this difference was not tested for statistical significancy. Our findings show lower levels of sensitivities for the Gram stains as compared to other studies ${ }^{12,17}$. In most studies, India ink has been reported to have a lower sensitivity rate compared to Gram stain and demands greater levels of expertise than the Gram stain ${ }^{11,12}$. Although subjective, a study conducted in Durban, South Africa by Coovadia et al. ${ }^{12}$, reported the outcomes of a brief interview held with a number of laboratory technologists; the consensus was that the Gram 
stain was much easier to perform and interpret than the India ink test. Bottone was the first to describe the role of the Gram stain for the diagnosis of cryptococcal infection in purulent exudates ${ }^{18}$ in his paper, Bottone described yeast as round lipoid bodies or stippled unicellular structures. Subsequent research work, although rather limited, further established that the Gram stain identified 50\% more cases of CM-positives than the India ink stain ${ }^{19}$. Other studies have reported that the Gram stain has a high sensitivity, ranging from $81 \%$ to $85 \%$ with regards to CM-positivity ${ }^{14,17}$. Despite all of these findings, it is surprising that most developing countries, including Malawi, still use India ink as the main diagnostic test for $\mathrm{CM}^{12}$.

Our study has established that LFA tests are the most accurate for diagnosing CM in HIV patients as compared with microscopic tests (Gram stain and India ink), with a sensitivity of $100 \%$ and a specificity of $98 \%$. This finding is in agreement with several studies conducted around the world $8,10,11,14,20-22$. As highlighted already, much of the diagnosis of CM is relatively simple and can be associated with a range of techniques: direct visualization, histopathology, culture and the detection of cryptococcal antigen ( $\mathrm{CrAg}$ ) in bodily fluids ${ }^{23,24}$. It is evident that LFA is emerging as the best of these methods. Data from the manufacturers indicate that the sensitivity, specificity, positive predictive value and negative predictive value of the LFA is $99.5 \%, 99 \%, 98 \%$ and $99.7 \%$, respectively, using serum, plasma, CSF or urine, when compared with the culture method ${ }^{23}$. The LFA is associated with a range of advantages. For example, no pretreatment is required for samples; and the LFA shows high sensitivity for $\mathrm{CrAg}$ of all serotypes. Furthermore, the LFA is suitable for use in settings with no, minimal or advanced infrastructure. Results can be obtained rapidly (within 10 minutes) and with low overall costs. Finally, LFAs only require a minimal level of expertise as they are designed to be used at point of care (POC) where most cadres of health care staff can be able to run them.

The CrAg LFA has been designed to address the needs of low-income countries. It also meets the World Health Organization (WHO) ASSURED criteria for diagnostic tests (Affordable: low cost; Sensitive: equal to or to better than other CrAg tests; Specific: similar to other CrAg tests; User friendly: in a format similar to the pregnancy test strip; Rapid/robust: produces a clear result in 10 minutes; Equipment-free: the test requires no electricity or laboratory infrastructure; and Delivered: the test is small, lightweight, requires no refrigeration and has a long shelf life) ${ }^{8,20,23}$. This therefore means that LFAs are also useful because they do not require invasive procedures in order to obtain the samples for assay. As such, there is a need for governments and policy makers among low-resource countries to consider the inclusion of LFAs in routine CM diagnosis in their health care systems. This has a critical potential to improve patient management and hence reduce the mortality rate associated with CM.

This research was associated with some limitations which need to be taken into consideration. First, this study was conducted at a central hospital; experienced medical technologists and technicians interpreted both the India ink and Gram stains. It cannot be assumed that the Gram stain will perform equally well in smaller peripheral laboratories with less experienced clinical laboratory technologists/ technicians reading the slides. Secondly, there was no blinding of results in this study; this may affect the results arising from the Gram stain and India ink tests, since they are more subjective. However, the technicians used in the present study were not aware of the intention to determine sensitivity and specificity; hence we believe that the tests were interpreted in the way that they would be interpreted in a routine clinical setting.

\section{Conclusion}

Our research found that the Gram stain was more sensitive than the India ink stain for the detection of CM. However, LFA was clearly the best method for diagnosing CM, and showed the highest levels of sensitivity and specificity. They also outperform cell culture, the current gold standard. LFA diagnostic methods have the potential to double the detection rate of CM-positive patients in resource-limited countries such as Malawi.

As such, LFAs may be considered to be the main diagnostic tests used for CM diagnostics in these countries

\section{Acknowledgements}

We would like to thank all of the clinical and nursing staff involved in the recruitment of participants and the laboratory technicians who conducted the tests.

\section{Authors' contributions}

Study conception: Master Chisale, Dikani Salema, Pocha Kamudumuli; study design: Master Chisale, Dikani Salema, Frank Sinyiza, Judith Mkwaila, Pocha Kamudumuli, Hsin-yi Lee.; data collection: Master Chisale, Dikani Salema, Frank Sinyiza, Judith Mkwaila, Pocha Kamudumuli, Hsin-yi Lee; data analysis: Master Chisale and Hsin-yi Lee.; manuscript preparation: Master Chisale, Dikani Salema, Frank Sinyiza, Judith Mkwaila, Pocha Kamudumuli, Hsin-yi Lee. All the authors contributed adequately towards the completion of this study. All authors read and approved the manuscript.

\section{Funding}

Funding was obtained from the Pingtung Christian Hospital (PTCH) Research Capacity Building Program, grant number: PS-IR-106001 and PS-IR-107001. The funders had no role in study design, data collection and analysis, or preparation of the manuscript.

\section{Availability of data and materials}

All data related to this study can be accessed at Mzuzu Central hospital upon consent given from the authorities

\section{Competing interests}

None declared.

\section{References}

1. Rajasingham R, Smith RM, Park BJ, Jarvis JN, Govender NP, Chiller $\mathrm{TM}$, et al. Global burden of disease of HIV-associated cryptococcal meningitis: an updated analysis. Lancet Infect Dis. 2017;17(8):873-81.

2. Veltman JA, Bristow CC, Klausner JD. Meningitis in HIV-positive patients in sub-Saharan Africa: A review. J Int AIDS Soc. 2014;17:19184.

3. Kambugu A, Meya DB, Rhein J, O'Brien M, Janoff EN, Ronald AR, et al. Outcomes of cryptococcal meningitis in Uganda before and after the availability of highly active antiretroviral therapy. Clin Infect Dis. 2008;46(11):1694-701.

4. Lessells RJ, P. C. Mutevedzi PC, Heller T, Newell MLN. Poor longterm outcomes for cryptococcal meningitis in rural South Africa. Vol. 101, SAMJ S Afr Med J. 2011;101:251-2.

5. Rothe C, Sloan DJ, Goodson P, Chikafa J, Mukaka M, Denis B, 
et al. A prospective longitudinal study of the clinical outcomes from cryptococcal meningitis following treatment induction with $800 \mathrm{mg}$ oral fluconazole in Blantyre, Malawi. PLoS One. 2013;8(6):e67311.

6. Gaskell KM, Rothe C, Gnanadurai R, Goodson P, Jassi C, Heyderman $\mathrm{RS}$, et al. A prospective study of mortality from cryptococcal meningitis following treatment induction with $1200 \mathrm{mg}$ oral fluconazole in Blantyre, Malawi. PLoS One. 2014;9(11):e110285

7. Rakhmanova AG, Giaurgieva OK. Clinical course of cryptococcosis in HIV infection. Klin Med (Mosk). 1999;77(1):39-42.

8. Williams DA, Kiiza T, Kwizera R, Kiggundu R, Velamakanni S, Meya DB, et al. Evaluation of fingerstick cryptococcal antigen lateral flow assay in HIV-infected persons: a diagnostic accuracy study. Clin Infect Dis. 2015;61(3):464-7.

9. Kauffman CA, Bergman AG, Severance PJ, McClatchey KD. Detection of cryptococcal antigen. Comparison of two latex agglutination tests. Am J Clin Pathol. 1981;75(1):106-9.

10. Tanner DC, Weinstein MP, Fedorciw B, Joho KL, Thorpe JJ, Reller LB. Comparison of commercial kits for detection of cryptococcal antigen. J Clin Microbiol. 1994;32(7):1680-4.

11. Cohen J. Comparison of the sensitivity of three methods for the rapid identification of Cryptococcus neoformans. J Clin Pathol. 1984;37(3):332-4.

12. Coovadia Y, Mahomed S, Dorasamy A, Chang C. A comparative evaluation of the Gram stain and India ink stain for the rapid diagnosis of cryptococcal meningitis in HIV infected patients in Durban. South African J Infect Dis. 2015;30(2):61-3.

13. National Statistical Office M. Preliminary Report for 2018 National census. Malawi Natl Stat Off [Internet]. 2018;39(1):32-8 [cited 2019 Jun 4]. Available from: https://malawi.unfpa.org/sites/default/files/ resource-pdf/2018 Census Preliminary Report.pdf.

14. WHO. Laboratory-based evaluation of 19 commercially available rapid diagnostic tests for tuberculosis. 2008 [cited 2019 Dec 4]. Available from: https://www.who.int/tdr/publications/documents/ diagnostic-evaluation-2.pdf.
15.MedCalc. Easy-to-use statistical software [Internet]. 2019 [cited 2019 Jun 4]. Available from: https://www.medcalc.org/calc/diagnostic test.php.

16. Coovadia YM, Solwa Z. Sensitivity and specificity of a latex agglutination test for detection of cryptococcal antigen in meningitis. $S$ Afr Med J. 1987;71:510-2.

17. Sato Y, Osabe S, Kuno H, Kaji M, Oizumi K. Rapid diagnosis of cryptococcal meningitis by microscopic examination of centrifuged cerebrospinal fluid sediment. J Neurol Sci. 1999;164(1):72-5.

18. Bottone EJ. Cryptococcus neoformans: Pitfalls in diagnosis through evaluation of gram-stained smears of purulent exudates. J Clin Microbiol. 1980;12(6):790-1.

19. Dunbar SA, Eason RA, Musher DM, Clarridge JE. Microscopic examination and broth culture of cerebrospinal fluid in diagnosis of meningitis. J Clin Microbiol. 1998;36(6):1617-20.

20. Johnman C. Laboratory diagnosis of HSV. J Fam Plan Reprod Heal Care. 2004;30(3):195.

21. McMullan BJ, Halliday C, Sorrell TC, Judd D, Sleiman S, Marriott $\mathrm{D}$, et al. Clinical utility of the cryptococcal antigen lateral flow assay in a diagnostic mycology laboratory. PLoS One. 2012;7(11):e49541.

22. Jarvis JN, Harrison TS, Lawn SD, Meintjes G, Wood R, Cleary S. Cost effectiveness of cryptococcal antigen screening as a strategy to prevent HIV-associated cryptococcal meningitis in South Africa. PLoS One. 2013;8(7):e69288.

23. Kumari S, Verma RK, Singh DP, Yadav R. Comparison of antigen detection and nested PCR in CSF samples of HIV positive and negative patients with suspected cryptococcal meningitis in a tertiary care hospital. J Clin Diagn Res. 2016;10(4):DC12-5.

24. Vidal JE, Boulware DR. Lateral flow assay for cryptococcal antigen: an important advance to improve the continum of HIV care and reduce cryptococcal meningitis-related mortality. Rev Inst Med Trop Sao Paulo. 2015;57(1):38-45. 